\title{
Plasma Thrombin-Activatable Fibrinolytic Inhibitor (TAFI) among Healthy Subjects and Patients with Vascular Diseases: A Validation Study
}

\author{
J. Monasterioa, P. Bermúdeza , D. Quirogaa , E. Franciscoª, B. Menesesa, \\ J. Montanerb
}

aVascular Biology and Haemostasis Research Unit, Universitary Hospital Vall d'Hebron, Barcelona, Spain bNeurovascular Research Laboratory, Universitary Hospital Vall d'Hebron, Barcelona, Spain

\section{Key words}

TAFI - Ischemic stroke · TPA · Thrombolysis .

Recanalization resistance

\begin{abstract}
Thrombin activable fibrinolysis inhibitor antigen levels (TAFI Ag ) exhibit a great inter-individual variability in healthy populations. Our aim is to determine whether variability is due to physiologic variations depending on genetic control or due to validation of the method, in order to allow a better interpretation of the results in patients with vascular diseases. With this purpose, we performed a strategy validation of specific ELISA method, Zymutest TAFI Ag Hyphen Biomed, based on a commercial monoclonal antibody. After methodology validation we have recently determined plasma TAFI Ag levels in several groups of diseases such as septic patients, menopause and cerebrovascular diseases. TAFI was finally determined in acute ischemic stroke to know its relationship with stroke evolution and response to thrombolytic treatments.
\end{abstract}

Copyright $\subset$ 200e S. Karger AG, Basel

\section{KARGER}

Fax +41613061234

E-Mail: karger@karger.ch www.karger.com
(C) 2004 S. Karger AG, Basel

1424-8832/04/0336-0382\$21.0/0

Accessible online at: www.krager.com/pht

\section{Introduction}

Thrombin-activable fibrinolysis inhibitor (TAFI), also known as procarboxypeptidase $\mathrm{B}$, is a plasma zymogen that potently inhibits fibrinolysis [1] when converted to an enzyme. The direct action of TAFI as an inhibitor of clot lysis involves removal of carboxy-terminal lysyl and arginyl residues from partially degraded fibrin [2]. Consequently, plasminogen binding sites are eliminated and plasminogen activation and fibrinolysis are inhibited [3]. Accordingly, it has been hypothesized that increased TAFI activity is associated with a proneness to thrombosis.

In fact, elevated TAFI plasma levels have been found in men with symptomatic coronary artery disease [4] and are a mild risk factor for deep vein thrombosis [5]. Recently, high levels of TAFI has also been found in stroke patients [6], a field in which TAFI over expression might block spontaneous or therapeutic thrombus resolution.

The beneficial effect of tPA, has been related to early brain tissue reperfusion and arterial recanalization, leading to reduced infarct size and better clinical outcome. Any acquired resistance to tPA as that potentially exerted by TAFI might reduce the benefit of thrombolysis.

In this study, we performed a strategy validation of an specific ELISA method (Zymutest TAFI Ag Hyphen Biomed) based on a commercial monoclonal antibody. Moreover, after methodology validation we have deter-

Dr. Jasone Monasterio, Unidad de Recerca de Biología Vascular y Hemostasia. Hospital General Vall d'Hebrón

Pg. Vall d'Hebron 119-129

08035. Barcelona. Spain

Tel/Fax: +34 932746 234; E-mail: 5877jma@comb.es 
mined plasma TAFI Ag levels in several groups of diseases such as septic patients, menopause and cerebrovascular diseases. Total TAFI Ag was determined using this validated method in acute ischemic stroke to study its relationship with thrombolytic treatment response, studying TAFI association with the rate of cerebral arteries recanalization following tPA infusion.

\section{Material and Methods}

Zymutest TAFI Ag, Hyphen-BioMed assay measures total TAFI Ag in plasma: pro-TAFI, active TAFIa and TAFIai coupled to inhibitor . The validation of the assay was carried out taking into account different pre-analytical and analytical variables which may affect the results and which are herewith described. To determine the influence of the anticoagulant used for collection from the plasma, EDTA and Citrate samples from the same subjects were analyzed for TAFI measurement. The samples were prepared at room temperature and the incubations were made at $37 \mathrm{oC}$. The intra and inter-assay $\mathrm{CV}$ was calculated for reproducibility using normal lyophilized plasma (citrol DadeBehring) and the determination was repeated six times, distributed at different points of the plate. The specificity for the determination of TAFI Ag in plasma was established by Dr. J.A. from Hyphen BioMed. A recovery study was performed. The linearity of the calibration curve was proven using a calibration standard with assigned values with the calculation of the regression coefficient of different concentrations of the calibration curve. Pro-zone effect and precision were evaluated using two controls included in the commercial kits: C1 and C2. Lastly, the sensitivity of the method was determined by taking the lowest concentration of the calibration curve making greater serial dilutions and calculating the minimum detection limit. Target dilution was calculated as well as TAFI values distribution (KolmogorovSmirnov).

\section{Study Population}

A group of healthy male and female donors $(n=81)$ from among the hospital staff and blood bank donors with a mean age of 50.3 years (range from 18 to 81 years) was studied. A group of patients from the intensive care unit (ICU) with sepsis and multi organic failure (MOF) $(n=27 ; 19$ males and 8 females aged from 51 to 79 years) versus a control group from the ICU without sepsis or MOF $(n=21 ; 11$ males and 10 females, age ranging from 31 to 73 years) was studied. Also,15 women in fertile period versus 12 women in menopause were studied prior to initiating hormonal replacement therapy (HRT) and after six months receiving

(TAFI) among Healthy Subjects and Patients with Vascular Diseases
HRT. The results were compared with those of a control group of healthy women with regular menstruation.

Finally, in a prospective study we included a group of consecutive patients with acute ischemic stroke $(n=66)$ confirmed by a neurologist, expert in neurovascular diseases, with samples being taken on admission (baseline, within first 24 hours of symptom onset). Another group of patients in the chronic phase of ischemic stroke $(n=30)$ was also recruited. Patients with cancer and inflammatory diseases were excluded.

In this preliminary report we studied 45 patients with acute cardio embolic ischemic stroke, admitted to hospital within the first 3 hours after stroke symptoms onset, with a Transcraneal Doppler documented Middle Cerebral Artery occlusion who were treated with a tPA standard dose of 0.9 $\mathrm{mg} / \mathrm{kg}$ distributed in as $10 \%$ in bolus and $90 \%$ in continuous perfusion during one hour. TCD examination was performed before treatment with tPA and by the end of tPA infusion, and again at $6 \mathrm{~h}$ and $12 \mathrm{~h}$ after stroke symptoms onset. Pre-treatment TAFI Ag was determined to study its relation to cerebral artery recanalization.

\section{Statistical Analysis}

Statistical analysis was carried out with the SPSS statistical package version 10.0. The results of quantitative variables were expressed in means with standard deviation (SD). The Students t test was used to evaluate the differences between groups considering values of $p<0.05$ as statistically significant. TAFI values were normally distributed (Kolmogorov-Smirnov and P-P plot).

\section{Results}

\section{Method Validation}

Validation of the anticoagulant was made to determine the influence of the anticoagulant EDTA and citrate on TAFI Ag concentrations in samples from the same healthy subjects $(\mathrm{n}=30)$. The TAFI Ag concentrations in citrate and EDTA were $X=115.29 \%$; $S=27.39 \%$ and $X=122.55 \%$; $\mathrm{SD}=24.5 \%$, respectively, with a good correlation between both anticoagulants, $(\mathrm{r}=0.82 ; \mathrm{p}=0.018)$ although citrate values were significantly lower. To demonstrate that this difference was not due to the type of anticoagulant but rather to the dilution factor corresponding to the sample extracted in citrate, we calculated the dilution factor of this anticoagulant on the sample when the proportion is one volume of citrate per nine of blood, the result of which was 1.08. On correcting the citrate values taking the dilution factor into account, no significant differences were found between the TAFI levels in citrate compared to the values found in 
EDTA ( $p=0.637$ ). This suggests that for TAFI determination samples may be taken regardless of which of the two anticoagulants had been used provided that the dilution factor corresponding to the citrate is applied. The optimum dilution recommended in the healthy population was of $1 / 50$. In patients with sepsis and stroke the target dilution was calculated resulting in an optimum dilution greater than or equal to $1 / 100$. Reproducibility results: Intra assay $\mathrm{CV}$ was 6.56 \% in 7 patients, with inter assay CV C-1 being $6.08 \%$ on 5 different days. Specificity of the assay: according to a personal communication of Dr. J. A. (Hyphen BioMed, France) the TAFI Ag test recognizes all the TAFI pro-activated, activated and inactivated forms. With respect to the recovery study in the standard used for the calibration curve, $100 \%$ of the expected value was recovered in the different high (154\%) and low concentrations (7.73 \%) coinciding with the concentration expected and found. Linearity was evaluated with the regression coefficient $(r=0.94)$ of different concentrations of the calibration curve (max. $148 \%$ and min. $7.4 \%$ ). No pro-zone effect or inhibition was observed since the dilutions were adjusted previously in search of the target dilution for each study group. We evaluated the precision of the method with two controls included in the commercial kit: C-1 (assigned value: $104 \%$; range 89-119 \%) with a value of $101 \%$, and C-2 (assigned value: $55 \%$; range 46-64 \%) with a value of $47 \%$. With respect to sensitivity, with greater dilutions of the samples with a concentration of $7 \%$, the sensitivity of the method corresponded to the lowest concentration of the calibration curve as the detection limit: No substances which may interfere like haemolysis or hyperlipidemia were observed in the samples studied. Provided that the expiry dates of the reagents and the preservation temperature are followed, no limitations were observed in regard to their suitability inter-assay C.V. As recommended by the manufacturer, samples that had been repeatedly frozen were not used.

\section{TAFI Level in the Healthy Population}

Following values were determined in the validation study of the total TAFI levels in a population of supposedly healthy donors $(n=81)$ with a mean age of 50.3 years (range: 18 - 82 years): $X=114.29 \%$; $\mathrm{SD}=29.62 \%$ with the range of normality being $55.05-173.53 \%$ and a maximum value of $174.27 \%$ and a minimum value of $54.71 \%$. On evaluation by age groups, each one comprising a decade, no significant differences were found among the groups $(p=0.117)$. Neither were significant differences found on evaluation of normality according to gender (males: $n=44 ; X=111.19 \%$; $\mathrm{SD}=29.72 \%$ with a mean age of 50.7 years, and in females: $\mathrm{n}=37 ; \mathrm{X}=118.29 \%$; $\mathrm{SD}=29.44 \%$ with a mean age of 50 years; $\mathrm{p}=0.284$ ). We have checked that TAFI Ag values are normally distributed in healthy population (KolmogorovSmirnov).

\section{TAFI Level in Patients with Vascular Diseases}

In the 27 patients from the ICU with septic shock and MOF, the antigen TAFI values found were $\mathrm{X}=193.43 \%$; $\mathrm{SD}=56 \%$ being $\mathrm{X}=117.8 \%$ : $\mathrm{SD}=48 \%$ in the 21 ICU patients without sepsis or MOF considered as the control group $(\mathrm{p}<0.01)$.

In the small group $(n=12)$ of women in menopause receiving HRT, the values before and after initiation of the treatment were: $\mathrm{X}=126.82 \% ; \mathrm{SD}=29.17 \%$ and $\mathrm{X}=123.95$ $\% ; \mathrm{SD}=24.83 \%$, respectively, and no differences were found $(\mathrm{p}=0.42)$.

In the group of 66 consecutive patients with acute ischemic stroke significantly higher TAFI Ag levels were found compared to the healthy control group [X=158.34\%; $\mathrm{SD}=52.46 \%$ and $\mathrm{X}=105.67 \%$; SD $30.25 \%$, respectively $(\mathrm{p}<0.001)]$. The same was found with the values of patients with chronic cerebral ischemic stroke $(X=234.45 \%$; $\mathrm{SD}=61.25 \%$ and $\mathrm{X}=105.67 \%$; $\mathrm{SD}=30.25 \%$; $\mathrm{p}<0.01)$. Nine patients who had received oral anticoagulants were excluded from this group.

According to classical cardiovascular risk factors, only dislipemia tended to be related to higher TAFI levels. No correlation was found between neurological status (baseline National Institutes of Health Stroke Scales scores) and TAFI levels.

Among stroke patients receiving tPA, no differences in baseline TAFI levels existed regarding age, gender and cardiovascular risk factors. Only hypertensive patients had higher baseline TAFI levels (168.77 $\pm 59.07 \%$ versus $133.54 \pm 26.97 \%$; $p=0.025)$. Surprisingly, we observed that patients who recanalized by the end of tPA infusion had significantly higher total circulating TAFI baseline levels as compared to those who did not recanalize (178.84 54.69 versus 141.84 45.69\%; $\mathrm{p}<0.05$ ). Our results also showed that, for patients with complete recanalization during tPA infusion, TAFI values came down to values similar to those of healthy controls.

\section{Discussion}

We have documented our current status of standardization of one TAFI antigen method before introducing a reference measurement system. This effort is made in view of the important role of TAFI in health and disease.

Among a healthy Mediterranean population we have shown TAFI distribution for gender and age groups. Because of the large inter-individual variability of TAFI antigen level and the weak relationship with lifestyle char- 
acteristics [7] it has been suggested that TAFI antigen is mainly under genetic control [8].

Our findings in ICU patients [9] suggests that TAFI may play an important role in the mechanism of organ failure associated sepsis. However, others have reported that TAFI activity in plasma was significantly low in patients with infection and in those with organ failure, suggesting that this might be due to enhanced consumption [10]. Recently it has been shown that TAFI 325 variants affect the outcome of meningococcal disease [11]. Altogether these results suggests that a combined approach measuring TAFI levels and determining TAFI genotypes will be needed in future human experiments [12].

Among menopausal women receiving HRT we observed no change in TAFI level, however it is possible that longer or alternative regimes to that used here may effectively reduce TAFI levels in a protection manner [13].

Regarding stroke patients, it has been demonstrated that TAFI is over expressed after ischemic stroke of any etiology [14], and TAFI antigen and activity respectively have been proposed as a risk factor for stroke [15].

These results are interesting because beside antiplatelet drugs the new oral direct inhibitors of thrombin might play an important role in the treatment and prevention of stroke. Our study also shows, paradoxically, that the lowest TAFI values measured before the administration of thrombolytic therapy for ischemic stroke are related to resistance of tPA induced recanalization of cerebral artery, reducing the success of this therapy. We hypothesize that when TAFI is activated it gets fixed to the clot surface to inhibit t-PA action. Therefore a "consumption" of total TAFI antigen might exist.

An alternative explanation, based on recent studies about TAFI polymorphisms, is that point mutations in TAFI gene may generate structural changes in the TAFI molecule, that makes difficult its recognition by the commercial antibody and increases the antifibrinolytic properties of TAFI.

Another important conclusion of our study is that cerebral vessel reopening leads to total TAFI Ag level normalization, indicating that brain hypoxia is the stimulus for TAFI over- expression.

Our results may suggest that blockage of TAFI activation or inhibition of activate TAFI could be assayed as synergistic adjuvant in thrombolytic therapy, although direct thrombin drugs could also be an attractive option for inhibiting TAFI activation. In fact, inhibition of TAFI has shown to accelerate tPA-induced clot lysis in vitro and to enhance thrombolysis in animal models [16,17].

In the future, TAFI measurement might help to optimise treatments in vascular patients. However, we should take into account that other fibrinolysis inhibitors such as PAI-1 [18] may also exert potent effects, therefore a global test will be desirable to guide clinical decisions.

\section{References}

1 Bajzar L, Manuel R, Nesheim ME. Purification and characterization of TAFI, a thrombin-activable fibrinolysis inhibitor. J Biol Chem 1995;270:14477-14484.

-2 Wang W, Boffa MB, Bajzar L, Walker JB Nesheim ME. A study of the mechanism of inhibition of fibrinolysis by activated thrombin-activable fibrinolysis inhibitor. J Biol Chem 1998;273:27176-27181.

-3 Mosnier LO, von deM Borne PA, Meijers JO, Bouma BN. Plasma TAFI levels influence the clot lysis time in healthy individuals in the presence of an intact intrinsic pathway of Coagulation. Thromb Haemost 1998;80:829-835.

$\checkmark 4$ Silveira A, Schatteman K, Goossens F, Moor E, Scharpe S, Stromqvist M, Hendriks D, Hamsten A. Plasma procarboxypeptidase $\mathrm{U}$ in men with symptomatic coronary artery disease. Thromb Haemost 2000;84:364368. van Tilburg NH, Rosendaal FR, Bertina RM. Thrombin activatable fibrinolysis inhibitor and the risk for deep vein thrombosis. Blood. 2000;95:2855-2859.

Montaner J, Ribó M, Monasterio J, Molina CA, Álvarez-Sabín J. High thrombinactivable fibrinolysis inhibitor [TAFI] levels in the acute phase of ischemic stroke. Stroke 2003;34: 1038-1040.

Chetaille P, Alessi Mc, Konassi D. Plasma TAFI Ag Variations in Healthy Subjects. Thromb Haemost 2000;83:902-905.

8 Henry M, Augbert H. Morange PE et al. Identification of polymorphisms in the promoter and the 3' region of the TAFI gene: evidence that Plasma TAFI antigen levels are strongly genetically controlled. Blood 2001;97:2053-2058.
9 Francisco E, Ruiz-Rodríguez JC, Caballero J, Monasterio J, Bóveda JL. ThrombinActivatable Fibrinolysis Inhibitor (TAFI) Phenotype In Severe Sepsis. 15 th Annual Congress of the European society of intensive care medicine. 2002.

10 Watanabe $\mathrm{R}$, Wada $\mathrm{H}$, Watanabe $\mathrm{Y}$, Sakakura M, Nakasaki T, Mori Y, Nishikawa M, Gabazza EC, Nobori T, Shiku H. Activity and antigen levels of thrombinactivatable fibrinolysis inhibitor in plasma of patients with disseminated intravascular coagulation.Thromb Res 2001;104:1-6.

11 Kremer Hovinga JA, Franco RF, Zago MA Ten Cate H, Westendorp RG, Reitsma PH. A functional single nucleotide polymorphism in the thrombin-activatable fibrinolysis inhibitor (TAFI) gene associates with outcome of meningococcal disease. J Thromb Haemost 2004;2:54-57. 
12 Guimaräes AHC, Van Tilburg NH, Vos HL, Bertina RM and Rijken DC. Association between thrombin activable fibrinolysis inhibitor genotype and levels in plasma: Comparison of different assays. $\mathrm{Br} \mathrm{J}$ Haematol 2004;124:659-665.

13 Vogelvang TE, Leurs JR, van der Mooren MJ, Mijatovic V, Hendriks DF, Neele SJ, Netelenbos JC, Kenemans P. Raloxifene reduces procarboxypeptidase $U$, an antifibrinolytic marker. A 2-year randomized, placebo-controlled study in healthy early postmenopausal women. Menopause 2004;11: 110-115.
14 Montaner J, Ribó M, Molina C, Monasterio J, Arenillas J, Delgado P, Pujadas F, Alvarez-Sabín J. Thrombin-Activable Fibrinolysis Inhibitor (TAFI) level is related to t-PA induced recanalization resistance after human ischemic stroke. Stroke 2003;34:240.

15 Santamaria A, Oliver A, Borrell M, Mateo J, Belvis R, Marti-Fabregas J, Ortin R, Tirado I, Souto JC, Fontcuberta J. Risk of ischemic stroke associated with functional thrombinactivatable fibrinolysis inhibitor plasma levels. Stroke 2003;34:2387-2391.

16 Mutch NJ, Moore NR; Wang E et al. Thrombus lysis by uPA, scuPA, and tPA is regulated by plasma TAFI. J Thromb Haemost 2003;1:2000-2007.
17 Nagashima M, Werner M, Wang M. An inhibitor of activated thrombin-Activatable Fibrinolysis inhibitor potentiates TissueType plasminogen Activator-induced thrombolysis in an Rabbit jugular vein thrombolysis model. Thromb Res 2000;333-342.

18 Ribó M, Montaner J, Molina CA, Arenillas JF, Rubiera M, Monasterio J, Huertas R, Santamarina E, Maurino J, Alvarez-Sabín J. Baseline plasma PAI-1 level predicts recanalization, hemorrhagic transformation, and outcome in tPA-treated patients. Stroke 2004;35:292. 